\title{
Abdominal Panniculectomy
}

National Cancer Institute

\section{Source}

National Cancer Institute. Abdominal Panniculectomy. NCI Thesaurus. Code C51597.

Surgical removal of excess skin and adipose tissue from the abdomen. 